\title{
Virulencia, producción y desplazamiento de nematodos entomopatógenos sobre larvas del picudo de la guayaba Conotrachelus psidii Marshall (Coleoptera: Curculionidae) en laboratorio
}

\author{
Yalexy Delgado-Ochica ${ }^{1 凶}$, Adriana Sáenz Aponte ${ }^{1}$
}

Received: 01-10-2012 Accepted: 11-11-2012 Published on line: 27-11-2012

Edited by Alberto Acosta

Virulence, production and dispersal of entomo-
pathogenic nematodes to larvae of the guava wee-
vil Conotrachelus psidii Marshall (Coleoptera: Curculionidae) in the laboratory. Abstract. The guava weevil Conotrachelus psidii Marshall is a major pest affecting guava cultivation in Santander, Colombia; it causes serious losses in the quality and the volume of fruit produced. Biological control is a viable option for pest management; entomopathogenic nematodes (EPNs), particularly, have shown good results (63-90\% mortality) in controlling fourth instar larvae of the guava weevil. In this study we evaluated the effect of seven species of EPNs isolated in Colombia: Steinernema websteri JCL006, Steinernema sp. 1 JCL024, Steinernema sp. 2 JCL007, Steinernema sp. 3 JCL027, S. colombiense SNI0198, Heterorbabditis bacteriophora HNI0100 and Heterorbabditis sp. SL0708 on fourth instar larvae of the guava weevil in laboratory conditions, and measured the production and the displacement of the most virulent. Heterorbabditis sp. SL0708 induced mortality of 85\%, Steinernema sp. 1 JCL024 75\% and S. colombiense SNI0198 55\%, the other species of EPNs, less than $25 \%$ mortality. Increased production of JI by weevil larva was recorded in Heterorbabditis sp. SL0708, which also showed greater recognition capability when the host was C. psidii.

Keywords: Biological control, Heterorbabditis sp., juvenil infective, Steinernema sp.

1 Laboratorio de Control Biológico, Facultad de Ciencias, Pontificia Universidad Javeriana, Bogotá D.C., Colombia.
Resumen. El picudo de la guayaba Conotrachelus psidii Marshall es una de las principales plagas del cultivo de la guayaba en Santander - Colombia, causa pérdidas severas afectando la calidad y cantidad de los frutos. El control biológico es una opción viable para el manejo de la plaga y en especial, los nematodos entomopatógenos (NEPs) han mostrado buenos resultados (63-90\% mortalidad) para el control de larvas de cuarto instar del picudo de la guayaba. En el presente estudio se evaluó el efecto de siete especies de NEPs aislados en Colombia: Steinernema websteri JCL006, Steinernema sp. 1 JCL024, Steinernema sp. 2 JCL007, Steinernema sp. 3 JCL027, S. colombiense SNI0198, Heterorhabditis bacteriophora HNI0100 y Heterorhabditis sp. SL0708 sobre larvas de cuarto instar del picudo de la guayaba en condiciones de laboratorio. También se evaluó la producción y el desplazamiento de las especies de NEPs más virulentas. Se registró que Heterorhabditis sp. SL0708 genera una mortalidad del $85 \%$ sobre el picudo de la guayaba, Steinernema sp. 1 JCL024 del 75\% y S. colombiense SNI0198 del 55\%, las otras especies de NEPs generaron mortalidad menor al $25 \%$. La mayor producción de JI por larva del picudo se registró en Heterorbabditis sp. SL0708, la cual también mostró mayor capacidad de reconocimiento cuando el hospedero es $C$. psidii.

Resumo. O bicudo-da-goiaba Conotrachelus psidii Marshall, é uma das principais pragas do cultivo da goiaba no Sanander - Colômbia. Ocasiona perdas muito grandes, afetando a qualidade e a quantidade dos frutos. O controle biológico é uma opção viável para o manejo da praga, especialmente, os nematóides entomopatogênicos-(NEPs) mostraram bons resultados (63-90\% mortalidade) para o controle do quarto instar do bicudo-da-goiaba. No presente estudo foi avaliado o efeito de sete espécies de NEPs isolados na Colômbia: Steinernema websteri JCL006, Steinernema sp. 1 JCL024, Steinernema sp. 2 JCL007, Steinernema sp. 3 JCL027, S. colombiense SNI0198, Heterorhabditis bacteriophora HNI0100 e Heterorbabditis sp. SL0708, sobre larvas de quarto instar do picudo da goiaba no laboratório. A produção, e o deslocamento das espécies mais virulentas também foram medidos. Para Heterorhabditis sp. SL0708 foi registrada mortalidade de 85\%, para Steinernema sp. 1 JCL024 do 75\% e para S. colombiense SNI0198 do 55\%, as outras espécies de NEPs causaram mortalidade do inseto menor que $25 \%$. A maior produção de JI por larva do bicudo foi registrada em Heterorbabditis sp. SL0708, a qual também mostrou maior capacidade de reconhecimento quando o hospedeiro é C. psidii. 


\section{Introducción}

La mayor producción de guayaba en Colombia se encuentra en el Departamento de Santander con 51.176 toneladas $(34 \%)$ de las 145.665 toneladas a nivel nacional, seguido por Boyacá (20\%), y Tolima $(12,3 \%)$. A partir de la guayaba se sustenta la agroindustria del bocadillo, cuya producción anual en Santander se valora en más de US $\$ 24$ millones anules (1), sin embargo, su producción se ha visto afectada por la plaga conocida como el picudo de la guayaba Conotrachelus psidii Marshall (Coleoptera: Curculionidae). El daño es realizado por las larvas que se alimentan de las semillas y la pulpa, que provocan un ennegrecimiento y endurecimiento de la parte afectada. Las excretas de la larva en el interior de la guayaba ocasionan petrificación del fruto, maduración prematura y caída del mismo (2), haciendo al fruto inservible para cualquier forma de consumo y para la agroindustria del bocadillo.

El ciclo del picudo dura en promedio 199 días: huevo, 4 a 7 días; la larva pasa por cuatro instares larvales, del primero al tercero ocurren dentro del fruto, 42 a 56 días; el cuarto instar larval ocurre en el suelo, 60 a 90 días; pupa en el suelo, 30 a 60 días. Los adultos salen del suelo entre los 20 y 30 días después. La infestación en los frutos por parte de las larvas, se ha observado el valor más alto durante la cosecha, que va desde inicios de septiembre hasta diciembre $(1,3)$.

El control del picudo en Santander es muy escaso y difícil, dado que los guayabales se encuentran silvestres ó asociados a sistemas silvopastoriles, por lo que ésta plaga genera pérdidas económicas significativas y aumenta sus poblaciones en cada cosecha (1). Debido a las pérdidas provocadas, se buscan estrategias económicamente viables para el sistema agrícola de la región y ecológicamente sostenibles para el control del picudo, que puedan ser incorporadas a un programa de Manejo Integrado de Plagas (MIP) como el control biológico, control cultural, uso de feromonas, desarrollo de plantas resistentes, entre otras alternativas (4).

Uno de los agentes de control biológico son los nematodos entomopatógenos (NEPs) de las familias Steinernematidae y Heterorhabditidae (Rhabditida), los cuales son organismos patógenos obligados de insectos. Están asociados en un mutualismo con bacterias que causan septicemia y otras afecciones letales en el huésped. El ciclo de vida del nematodo tiene diferentes etapas de las cuales el Juvenil Infectivo (JI) es de vida libre que parasita al hospedero, al que busca mediante desplazamiento activo (crucero) o pasivo (emboscador). La asociación nematodo-bacteria es la que determina la efectividad y selectividad de estos controladores, razón por la cual se realizan gran variedad de ensayos en laboratorio para establecer la patogenicidad y virulencia de las especies de para seleccionar la especie a ser implementada en el control de un determinado organismo (5).

Los NEPs son potenciales agentes para el control del picudo de la guayaba dado que varios de sus estados ocurren en o sobre el suelo, lo que podría desencadenar una estrategia racional para manejar esta plaga, como se ha demostrado en otros estudios. Del Valle et al. $(5,6)$ y Dolinski et al. (7) demostraron la alta susceptibilidad del cuarto instar larval del picudo de la guayaba por parte de Heterorbabditis baujardii LPP7 (mortalidad 80\%) y Heterorhabditis indica Hom1 (mortalidad 85\%) en experimentos de laboratorio e invernadero a una dosis de 100 nematodos por larva del picudo de la guayaba (5-7).

Teniendo en cuenta la importancia del cultivo, los daños generados por el picudo y la posibilidad de los nematodos entomopatógenos para controlar esta plaga, se evaluó la virulencia de siete especies de NEPs aislados en Colombia, sobre larvas de cuarto instar del picudo. Además de la producción y desplazamiento de JIs de las especies más virulentas, bajo condiciones de laboratorio.

\section{Materiales y métodos}

Se evaluaron siete especies de NEPs (Tabla 1). De la 1 a la 6 son aislamientos obtenidos por CENICAFE, cuyos análisis de la secuencia de rDNA las clasificó como especies diferentes (8). La especie Heterorhabditis sp. SL0708 es un aislamiento realizado en la Pontificia Universidad Javeriana, cuyo ciclo de vida y patogenicidad, entre otras características, ha mostrado ser una especie distinta a Heterorbabditis bacteriophora (9)

La producción y la obtención de los JIs recién emergidos (sin fase de almacenamiento), de las diferentes especies de NEPs utilizadas en este estudio, 
se realizó a partir de larvas de último instar de Galleria mellonella (L.) (Lepidoptera: Pyralidae) con trampas White a $25^{\circ} \mathrm{C}$, siguiendo las recomendaciones de Sáenz y Olivares (10) y Realpe-Aranda (11).

Para la obtención de larvas de cuarto instar del picudo de la guayaba, se colectaron frutos infestados con la plaga provenientes de Guavatá (Santander). En el laboratorio se separaron las larvas de cuarto instar (longitud de 10-12 mm y coloración crema) y las larvas de otros instares se depositaron en bandejas plásticas con guayaba para permitir su desarrollo. Las larvas seleccionadas para los ensayos se dejaron 24 horas en observación antes de ser utilizadas, y se escogieron aquellas que se movían y presentaban coloración uniforme (sanas).

Pruebas de virulencia de JI: Se realizó un ensayo completamente al azar de ocho tratamientos; siete especies de NEPs y un control sin NEPs (agua destilada), con 10 replicas cada uno y una repetición en el tiempo, como se ha realizado en otros estudios (7). Cada unidad experimental consistió en una caja de petri de $3 \mathrm{~cm}$ de diámetro, con $16 \mathrm{~g}$ de arena de río estéril húmedo, se colocaron $100 \mathrm{JI}$ y una larva de cuarto instar del picudo de la guayaba con el volumen de agua para completar la capacidad del campo del sustrato $(30 \%$ de humedad P/P); las unidades experimentales contaban con una sola

Tabla 1. Especies y cepas de nematodos entomopatógenos evaluados para el control del picudo de la guayaba C. psidii.

\begin{tabular}{|c|c|c|c|c|c|}
\hline Especie & Cepa & $\begin{array}{c}\text { Municipio/ } \\
\text { Departamento }\end{array}$ & $\begin{array}{l}\text { Vegetación } \\
\text { asociada }\end{array}$ & $\begin{array}{c}\text { pH del } \\
\text { suelo }\end{array}$ & $\begin{array}{l}\text { \% contenido de } \\
\text { arcilla: limo: } \\
\text { arena }\end{array}$ \\
\hline Steinernema websteri & JCL006 & Chinchiná - Caldas & Bosque, café - guamo & $\begin{array}{l}3,5-5,2 \\
4,4-5,9\end{array}$ & $\begin{array}{l}10-17: 23-33: 54-66 \\
10-21: 14-24: 63-72\end{array}$ \\
\hline Steinernema sp. 1 & JCL024 & Buenavista - Quindio & Bosque, Café & $4,4-5,9$ & 10-21: 14-24: 63-72 \\
\hline Steinernema sp. 2 & JCL007 & Chinchiná - Caldas & Café - guamo & $\begin{array}{l}3,9-5,2 \\
4,4-5,9\end{array}$ & 10-17:23-33: 54-66 \\
\hline Steinernema sp. 3 & JCL027 & Sasaima -Cundinamarca & Plátano & 4,5 & 10-21:14-24: 63-72 \\
\hline Steinernema colombiense & SNI0198 & Quimbaya - Quindio & Cafetal & 4,9 & $35: 25: 40$ \\
\hline Heterorbabditis bacteriophora & HNI0100 & Fresno -Tolima & Sin datos & $\begin{array}{l}\text { Sin } \\
\text { datos }\end{array}$ & $\begin{array}{l}\text { Materia orgánica 5,9\% } \\
\text { Sin datos }\end{array}$ \\
\hline Heterorhabditis sp. & SL0708 & Alcalá - Valle del Cauca & Guadual & 4,7 & 12,8: 19,8: 68,7 \\
\hline
\end{tabular}

larva, dado que cuando estas son perturbadas, son bastante agresivas; se incubaron a $24^{\circ} \mathrm{C} \pm 1$. Se registró la mortalidad cada 24 horas durante 15 días. Las larvas del picudo muertas se disectaron para comprobar la presencia de nematodos.

Producción de JI a partir de larvas del picudo de la guayaba: Las especies de NEPs que generaron porcentajes de mortalidad superiores al $50 \%$, se les evaluó la producción de JI por larva de cuarto instar del picudo de la guayaba. Las larvas del picudo infectadas se incubaron a $25^{\circ} \mathrm{C}$ y después de 5 días, se montaron en trampas White modificadas $(11,12)$ para la recuperación de los JI. Los conteos de los JI se realizaron diariamente por dilución, siguiendo las recomendaciones de Kaya y Stock (12).

Búsqueda por el hospedero: Se usaron columnas de arena para evaluar la capacidad de los JI para desplazarse, encontrar y matar las larvas de cuarto instar del picudo de la guayaba, dado que en el suelo se profundizan a $10 \mathrm{~cm}$ en promedio. Se seleccionaron las especies de NEPs que presentaron la mayor virulencia $(+70 \%)$ y producción de JI.

Se utilizó un tubo de PVC de $10 \mathrm{~cm}$ de alto por $5 \mathrm{~cm}$ de diámetro, en el lado inferior se dejó una caja de petri pequeña ( $5 \mathrm{~cm}$ de diámetro). En el fondo de cada tubo, se ubicó una larva de $C$. psidii 
ó de G. mellonella inmovilizada con angeo metálico para impedir el movimiento vertical de la larva. Posteriormente se cubrió con sustrato estéril $(54 \%$ arena, 26\% arcilla, 19\% sedimentos, $\mathrm{pH}: 4,6 ; 3,4 \%$ materia orgánica; $40 \%$ humedad) proveniente de Guavatá $\left(05^{\circ} 55^{\prime} 09,2^{\prime \prime}\right.$ de latitud Norte y $73^{\circ} 41^{\prime}$ $37,5^{\prime \prime}$ de longitud Oeste; a $1.922 \mathrm{~m}$ de altitud) y sobre la columna se aplicó una de tres dosis de JI $(0,200$ o $500 \mathrm{JI}$ /tubo). La dosis de $200 \mathrm{JI}$ es la empleada en ensayos de efectividad de NEPs, mientras que la dosis de $500 \mathrm{JI}$ es cercana a $250.000 \mathrm{JI} / \mathrm{m}^{2}$, la cual es recomendada generalmente para aplicaciones en campo (13). Las columnas se taparon manteniendo la posición vertical y se incubaron por 72 horas a $25^{\circ} \mathrm{C}$. Se removieron las larvas muertas y se disectaron para verificar la presencia de nematodos. El diseño fue factorial de tres dosis, dos especies de nematodos y dos especies hospedero. Se realizaron 10 réplicas por tratamiento y se repitió el experimento una vez en el tiempo (a los 30 días).

Análisis estadístico: La mortalidad de cada ensayo fue convertida a porcentaje. Los datos que cumplían con los supuestos de normalidad y homogeneidad se les realizaron análisis de varianza de una vía (ANOVA); y los datos que no cumplían con los supuestos se les efectuaron la prueba de KruskalWallis $(\mathrm{H})$, con SPSS19. Los ensayos con diferencias significativas entre los tratamientos se analizaron con una prueba a posteriori de Tukey (paramétrico) ó de Dunnet (No-paramétrico) a una significancia de $\mathrm{p} \leq 0,05$, las diferencias se indican en las figuras con diferentes letras sobre las barras.

\section{Resultados}

Pruebas de virulencia de los JI: Se encontró que el picudo de la guayaba es susceptible a las siete especies de NEPs evaluadas. La especie Heterorhabditis sp. SL0708 presentó la mayor virulencia $(85 \pm 8 \%)$ seguida por Steinernema sp. 1 JCL024 $(78+8 \%)$ y $S$.

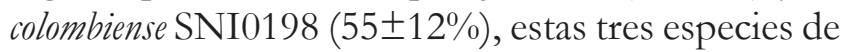
NEPs mostraron diferencias significativas $(\mathrm{p} \leq 0,05)$ con respecto al control $(0 \%)$ (Figura 1). Las otras especies de nematodos evaluadas Steinernema sp. 3 JCL027, H. bacteriophora HNI0100, S. websteri JCL006 y Steinernema sp. 2 JCL007 presentaron 25 a 15\% de mortalidad sobre las larvas del picudo. Respecto al tiempo, la mortalidad en el $80 \%$ de los tratamientos se registró en las primeras 48 horas, hasta las 72 horas.

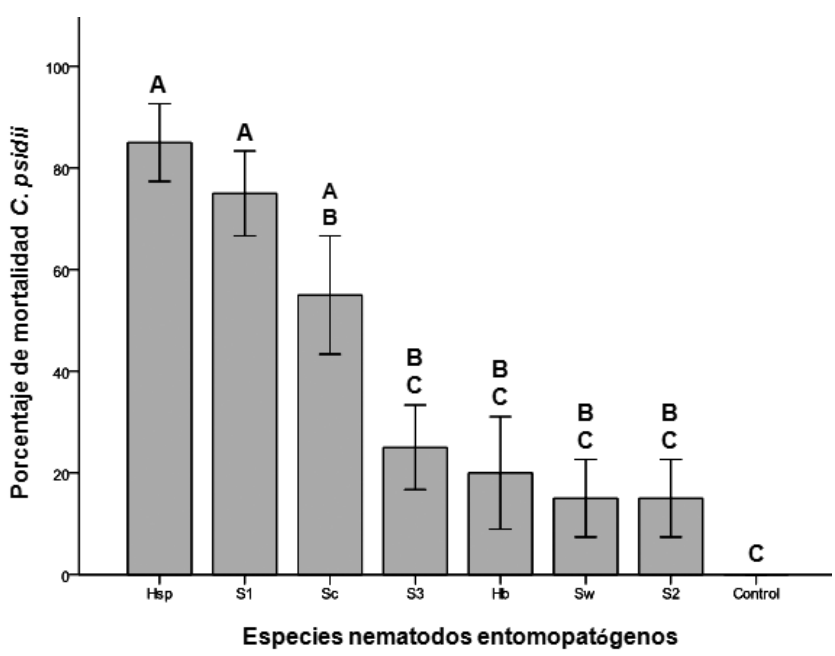

Fig. 1. Porcentaje de mortalidad ( $\pm E S$ ) de las larvas de cuarto instar del picudo de la guayaba (C. psidii) causada por la exposición a 100JI / larva de siete especies de nematodos entomopatógenos evaluados. Hsp: Heterorbabditis sp. SL0708; S1: Steinernema sp. 1 JCL024; Sc: Steinernema colombiense SNI0198; S3: Steinernema sp. 3 JCL027; Hb: Heterorbabditis bacteriophora HNI0100; Sw: Steinernema websteri JCL006; S2: Steinernema sp. 2 JCL007. Diferentes letras sobre las barras indican diferencias significativas (Dunnet, $\mathrm{p} \leq 0,05$ ).

Producción de JI por larva del picudo de guayaba: Heterorhabditis sp. SL0708, Steinernema sp. 1 JCL024 y $S$. colombiense SNI0198 fueron las especies que presentaron porcentajes de mortalidad superiores al

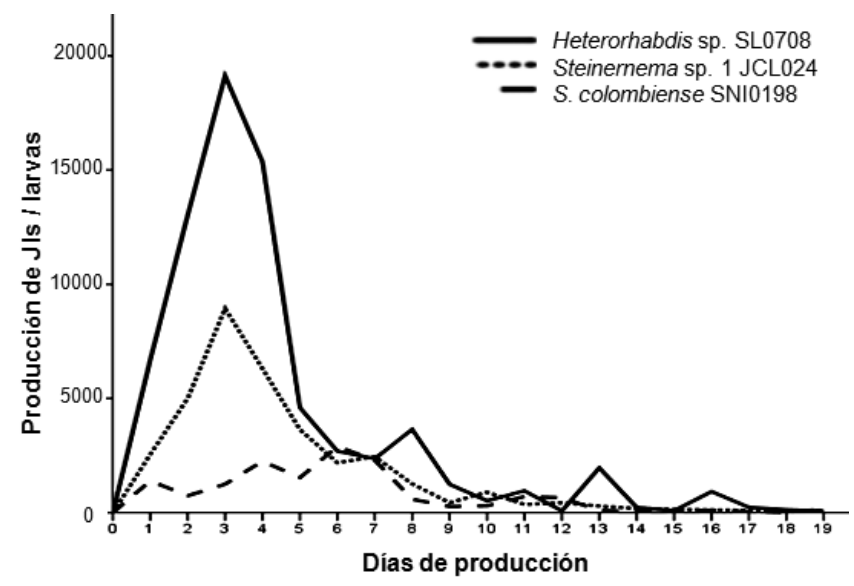

Fig. 2. Producción de JI de tres especies de nematodos entomopatógenos por larva de cuarto instar del picudo de la guayaba C. psidii en el tiempo. 
$50 \%$, sobre las cuales se evaluó la producción de JI/ larva del picudo de la guayaba. La mayor producción se registró al tercer día, y el 80\% de producción de los JI se alcanzó en los primeros ocho días, con incremento en la producción algunos días después en las tres especies evaluadas (Figura 2).

Heterorhabditis sp. SL0708 fue la especie que produjo el mayor número promedio de JI por

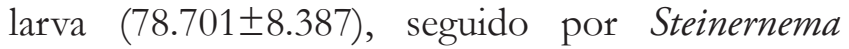
sp. 1 JCL024 (42.924 \pm 7.319$)$ y S. colombiense SNI0198 (24.879 5 5.885) (Figura 3). Se presentaron diferencias estadísticas $(\mathrm{p} \leq 0,05)$ entre Heterorbabditis sp. SL0708 y las otras dos especies.

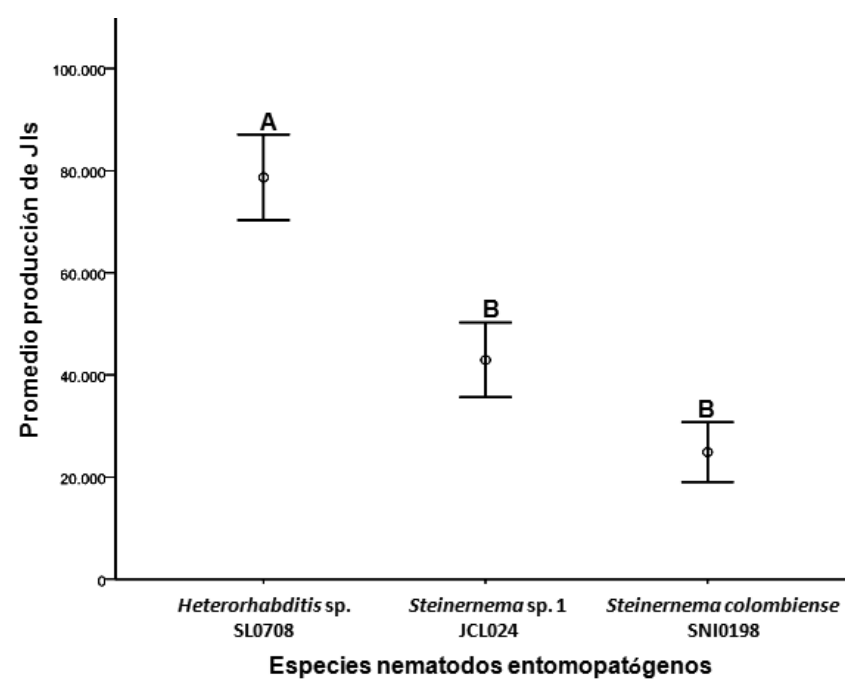

Fig. 3. Producción de JI ( $\pm \mathrm{ES})$ de tres especies de nematodos entomopatógenos por larva de cuarto instar del picudo de la guayaba C. psidii. Diferentes letras sobre las barras indican diferencias significativas (Tukey, $\mathrm{p} \leq 0,05$ ).

Búsqueda por el hospedero: Para las pruebas de las columnas de arena se evaluó Heterorhabditis sp. SL0708 y Steinernema sp. 1 JCL024 por presentar porcentajes de mortalidad superiores al $50 \%$. No se registraron diferencias significativas $(p \leq 0,05)$ entre los porcentajes de mortalidad de $C$. psidii y $G$. mellonella, pero si entre estos dos huéspedes con el control y entre las dosis ( $\mathrm{p} \leq 0,05)$ (Figura 4). El aislado Heterorhabditis sp. SL0708 presentó el mayor porcentaje de mortalidad sobre larvas del picudo de la guayaba $(75 \pm 11 \%)$ en la dosis de $200 \mathrm{JI}$; en la

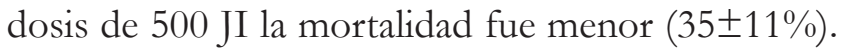
Steinernema sp. 1 JCL024 sobre larvas del picudo generó una mortalidad de $30 \pm 26-35 \pm 34 \%$, en las dosis de 200 JI e 500 JI, respectivamente. Para G. mellonella las dos especies de nematodos evaluadas mostraron mortalidad entre 75 y 35\% (dosis de 200 y $500 \mathrm{JI}$ respectivamente).

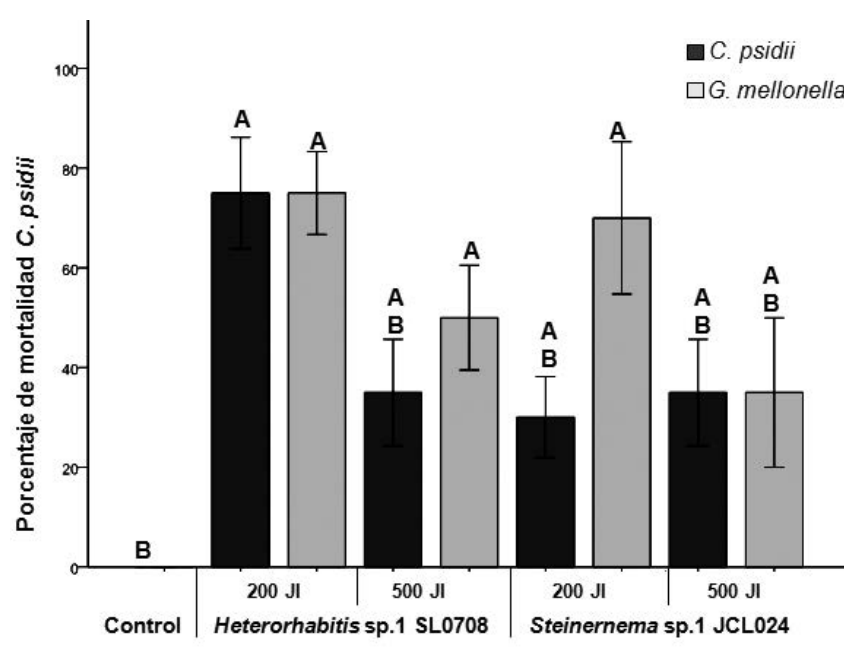

Fig. 4. Porcentaje de mortalidad ( $\pm E S$ ) de las larvas de cuarto instar del picudo de la guayaba (C. psidii) y de larvas de último instar de G. mellonella causada por Heterorbabditis sp. SL0708 y Steinernema sp. 1 JCL024 en dosis de 200 JI y $500 \mathrm{JI}$ en las columnas de arena. Diferentes letras sobre las barras indican diferencias significativas (Dunnet, $\mathrm{p} \leq 0,05$ ).

\section{Discusión}

En los ensayos de laboratorio se aplicaron $100 \mathrm{JI} /$ larva como se ha propuesto en la literatura (12), para evaluar la susceptibilidad de un hospedero y realizar el proceso de selección de la especie de NEP potencial para ser implementada como agente de control biológico. De las pruebas de laboratorio, las siete especies de nematodos evaluadas produjeron mortalidad larval, donde una especie de Heterorhabditis y una de Steinernema generaron mortalidades significativamente mayores a las otras especies. Dolinski et al. (14) evaluaron nueve especies de NEPs en larvas de cuarto instar del picudo de la guayaba, quienes plantean que todas las especies de Heterorbabditis evaluadas fueron más virulentas (80-75\%) que las especies de Steinernema (65-45\%), lo que difiere con el presente estudio, donde $H$. bacteriophora HNI0100 presentó 25\% mortalidad.

El tiempo de mortalidad registrado para todas las especies de NEPs evaluadas sobre C. psidii $(<72$ 
horas) fue menor en el presente estudio a la que se ha registrado para esta plaga, la cual, se encuentra en los primeros tres días para H. baujardi LPP7 (5), diez días para $H$. indica y 44 días para $S$. riobrave (14). El presente estudio se realizó con larvas recién emergidas del fruto, aproximadamente de seis semanas, mientras que en los estudios de Del Valle et al. (5) y Dolinski et al. (14) se realizaron con larvas que se encontraban al final de su cuarto instar larval, terminando sus ensayos en estado de prepupa. Estos resultados son coincidentes con lo registrado para el picudo de la nuez Curculio caryae (Coleoptera: Curculionidae), cuyas larvas más maduras parecen ser más resistentes a la infección de los NEPs que las larvas más jóvenes (15). En el caso de C. psidii, el cuarto instar larval es el estado en el cual abandona el fruto para profundizarse en el suelo, se vuelve inactivo y se convierte en prepupa; la prepupa es morfológicamente similar al cuarto instar larval, pero es un poco más pequeña, su cutícula pierde humedad y se vuelve más dura (16), por lo que el avance de sus estadios puede afectar posiblemente la capacidad de penetración de los NEPs.

La producción acumulada de los JIs para Heterorbabditis sp. SL0708 en larvas de G. mellonella es de 150.000 a $280.000 \mathrm{JI} /$ larva (10), estos valores son correspondientes a lo obtenido para $C$. psidii (74.071 JIs), teniendo en cuenta que éste representa un poco menos de la mitad del peso comparado con G. mellonella; para $H$. bacteriophora la producción acumulada es de $78.750 \mathrm{JI} /$ larva de $G$. mellonella y para S. colombiense es de $86.250 \mathrm{JI} /$ larva (11). Adicionalmente, estas producciones pueden ser muy variables, ya que oscilan entre 30.000 y 240.000 JI/larva dentro del mismo hospedero, esto depende principalmente de la capacidad del hospedero para suplir los requerimientos nutricionales de los nematodos (10). Estas producciones indican que Heterorhabditis sp. SL0708 tiene la capacidad de penetrar, matar y multiplicarse dentro del picudo de la guayaba, salir nuevamente al ambiente en busca de nuevos hospederos y probablemente persistir en el tiempo.

El tiempo de producción de los JIs de Heterorhabditis sp. SL0708, Steinernema sp. 1 JCL024 y S. colombiense SNI0198 a partir de larvas de C. psidii es similar a lo registrado en larvas de G. mellonella, donde la producción es mayor en los primeros 3 días y disminuye a través del tiempo, y durante los diez primeros días de emergencia se obtienen las mayores producciones de nematodos, con un 90\% de los JI (10 - 17). La producción de los JIs de Heterorhabditis sp. SL0708 inició a los 7-10 días después de la infección, por lo que se puede decir que éste nematodo presenta dentro del picudo de la guayaba un ciclo de vida largo y uno corto, según lo reportado en $G$. mellonella, en el cual se presenta un ciclo de vida largo y dos cortos (10).

Los ensayos en las columnas de arena permitieron evaluar la capacidad de búsqueda e infección de las especies de NEPs, registrando que Heterorbabditis sp. SL0708 tiene mayor capacidad que Steinernema sp. 1 JCL024 de encontrar y matar las larvas de C. psidii en la dosis de $200 \mathrm{JI}$. Los porcentajes de mortalidad no difieren entre las dos especies de nematodos cuando el hospedero es $G$. mellonella. Dolinski et al. (14) realizaron pruebas en columnas de arena para el picudo de la guayaba con dos especies de Heterorbabditis y una de Steinernema, donde los heterorhabditidos presentaron mayor mortalidad larval (70-80\%), lo cual coincide con el presente estudio; sin embargo, esos porcentajes de mortalidad se alcanzaron en la dosis de $500 \mathrm{JI}$ y en el presente estudio en la dosis de $200 \mathrm{JI}$. La dosis de $200 \mathrm{JI}$ corresponde a $1 \times 10^{9} \mathrm{JI} / \mathrm{ha}$, la cual es menor a $2,5 \times 10^{9} \mathrm{JI} /$ ha que se ha registrado en la mayoría de los casos para que los NEPs sean efectivos al ser aplicados al suelo $(18,19)$. En situaciones donde el hospedero es particularmente susceptible ó en condiciones controladas, tales como en laboratorio e invernadero, dosis menores también pueden ser efectivas (20).

Las dos especies de NEPs presentaron porcentajes de mortalidad elevados para G. mellonella (70-75\%) en las pruebas de las columnas de arena, lo que indica que las dos especies tienen la capacidad de desplazamiento vertical en $10 \mathrm{~cm}$ de profundidad, la cual es la distancia promedio en la que se encuentran las larvas de cuarto instar del picudo de la guayaba en el suelo. Este desplazamiento indica que las especies presentan estrategia de forrajeo tipo "crucero" (búsqueda activa), los cuales son más efectivos en encontrar el recurso sedentario y críptico que los tipo "emboscadores" $(21,22)$. La larva del picudo de la guayaba es inmóvil y permanece en el suelo por largos periodos de tiempo (60-90 días), por lo que presentar forrajeo tipo "crucero" por parte del NEP, es más efectiva para encontrar este hospedero. 
Aunque las dos especies de nematodos evaluadas tienen estrategia de forrajeo tipo "crucero", es Heterorbabditis sp. SL0708 la que genera mayor porcentaje de mortalidad para $C$. psidii. Se ha registrado que los NEPs presentan quimiorreceptores, con los cuales se orientan hacia su hospedero dependiendo de sus liberaciones de $\mathrm{CO} 2$, de otros olores como ácido láctico, heces, entre otros (21), y ese reconocimiento por un determinado hospedero va a afectar su desplazamiento y su eficacia, principalmente en los "crucero", ya que les confiere un mayor potencial para encontrarlo (22). La mayor virulencia registrada por Heterorbabditis sp. SL0708 indica que su capacidad para reconocer $C$. psidii es mayor que Steinernema sp. 1 JCL024.

Las pruebas de columnas de arena se realizaron con sustrato del área de estudio, el cual presentó materia orgánica, con mayor cantidad de arena (54\%) que de arcilla, donde la arena genera porosidad en el sustrato. La porosidad es un factor importante para los JIs dado que cuando los poros del suelo son más pequeños (poca arcilla-26\%) la movilidad de los JIs decrece y se reducen los niveles de oxígeno disponibles para los JIs, disminuyendo así su capacidad de supervivencia y de percibir las señales del hospedero (10 - 22, 23). Adicionalmente, las prácticas agrícolas como la aplicación de fertilizantes y plaguicidas también pueden afectar negativamente el comportamiento de los nematodos (22), por lo que la carencia de estos dos tipos de prácticas en el área guayabera santandereana y el tipo de suelo, favorece la aplicación de los NEPs.

\section{Conclusión}

Heterorhabditis sp. SL0708 fue el nematodo que presentó mayor virulencia, producción de JI por larva del picudo de la guayaba y capacidad para encontrar a C.psidii, es la especie que se debe evaluar en invernadero y campo para ser incorporada en programas de manejo integrado de plagas en Santander.

\section{Agradecimientos}

A la Pontificia Universidad Javeriana por la financiación del proyecto de investigación (4138); a Rosalva Velasco, Alvaro Hernández y German Rueda agricultores de
Guavatá (Santander) por el apoyo en la consecución del material biológico.

\section{Conflicto de intereses}

Los autores expresan que no existen más que intereses científicos alrededor de los resultados de esta investigación.

\section{Referencias}

1. Insuasty O, Monroy R, Díaz A, Bautista J. Manejo fitosanitario del cultivo de la guayaba (Psidium guajava L.) en Santander. Corpoica- ICA Imprenta nacional de Colombia. 2011, 40 p.

2. Insuasty O, Monroy R, Diaz A, Bautista J. Manejo integrado del picudo de la guayaba Conotrachelus psidii Marshall en Santander. Corpoica, Ica, Secretaria de Agricultura y desarrollo de Santander. 2007, 27 p.

3. Monroy E, Insuasty O. Aspectos biológicos y duración de los estadios del picudo de la guayaba Conotrachelus psidii. Revista Corpoica: Ciencia y Tecnología Agropecuaria 2006; 7 (2): 73-79.

4. Dolinski C, Lancely L. Microbial control of arthropod pests of tropical tree fruits. Neotropical Entomology 2007; 36 (2): 161-179.

5. Del Valle E, Dolinski C, Souza R, Samuels R. Performance de Heterorbabditis baujardi LPP7 (28) (Nematoda: Rhabditida) seleccionada para tolerancia a elevadas no controle de Conotrachelus psidii, (Coleoptera: Curculionidae). Nematología Brasileira 2005; 29: 199-205.

6. Del Valle E, Dolinski C, Barreto E, Souza R, Samuels R. Efficacy of Heterorhabditis banjardi LPP7 (Nematoda: Rhabditida) applied in Galleria mellonella (Lepidoptera: Pyralidae) insect cadavers to Conotrachelus psidii, (Coleoptera: Curculionidae) larvae. Biocontrol Science and Technology 2008; 18: 3341.

7. Dolinski C, Lancely L. Microbial control of arthropod pests of tropical tree fruits. Neotropical Entomology 2006; 36 (2): 161-179.

8. López-núñez JC, Cano L, Góngora CE, Stock P. Diversity and evolutionary relationships of entomopathogenic nematodes (Steinernematidae and Heterorhabditidae) from the Central Andean region of Colombia. Nematology 2007; 9 (3): 333341.

9. Sáenz AA, López JC. Ciclo de vida y patogenicidad del aislamiento nativo Heterorbabditis sp. SL0708 (Rabditida: Heterorhabditidae). Revista Colombiana de Entomología 2011; 37 (1): 43-47.

10. Sáenz AA, Olivares W. Capacidad de búsqueda de Steinernema sp. SNIO 198 (Rhabditida: Steinernematidae) para el control de Sagalassa 
valida Walker (Lepidoptera: Glyphipterygidae). Revista Colombiana de entomología 2008; 34 (1): 51-56.

11. Realpe-Aranda FJ, Bustillo-Pardey AE, LópezNúñez J.C. Optimización de la cría de Galleria mellonella (L.) para la producción de nematodos entomopatógenos parásitos de la broca del café. Cenicafé 2007; 58 (2): 142-157.

12. Kaya HK, Stock P. Techniques in insect nematology. In: Lacey L.A. (ed.). Manual of techniques in insect pathology. San Diego, CA. 1997; 281-324.

13. Shapiro-Ilan DI, Gouge DH, Piggott SJ, Patterson J. Application technology and environmental considerations for use of entomopathogenic nematodes in biological control. Comparative and General Pharmacology 2006; 38: 124-133.

14. Dolinski C, Del Valle EE, Stuart, R.J. Virulence of entomopathogenic nematodes to larvae of guava weevil, Conotrachelus psidii (Coleoptera: Curculionidae), in laboratory and greenhouse experiments. Biological control 2006; 38: 422-427.

15. Shapiro-Ilan DI, Mizell R, Campbell JF. Susceptibility of the plum curculio Conotrachelus nenuphar to entomopathogenic nematodes. Journal of Nematology 2002; 34 (3): 246-249.

16. Monroy E, Insuasty O. Aspectos biológicos y duración de los estadios del picudo de la guayaba Conotrachelus psidii. Revista Corpoica: Ciencia y Tecnología Agropecuaria 2006; 7 (2): 73-79.
17. Sáenz A, Luque J. Cultivo in vivo y método de almacenamiento para juveniles infectivos de Steinernema feltiae (Rhabditidae: Steinernematidae). Agronomia Colombiana 2000; 17 (1-3): 37-45.

18. Grewal PS, Ehlers RU, Shapiro-Ilan DI. Nematodes as biocontrol agents. CABI Publishing. New York. 2005, 505 p.

19. Wilson M, Gaugler R. Factors limiting short-term persistence of entomopathogenic nematodes. Journal Entomology 2004; 128 (4): 250-253.

20. Leite LG, Tavares FM, Goulart RM, Batista A, Parra JR. Patogenicidade de nematóides entomopatogênicos a larvas de $6^{\circ}$ instar do bicho-furão, Ecdytolopha aurantiana (Lepidoptera: Tortricidae), e avaliação de dosagens de Heterorbabditis indica na mortalidade do inseto. Revista Agrícola 2005; 80: 316-330.

21. Kaya HK, Gaugler R. Entomopathogenic nematodes. Annual Review of Entomology 1993; 38: 181-206.

22. Lewis EE. Behavioral ecology. In: Gaugler R. (Ed.). Entomopathogenic Nematology. CABI Publishing. New York. 2002, 205- 221.

23. Koppenhofer AM, Fuzy EM. Soil moisture effects on infectivity and persistence of the EN $S$. scarabaei, S. glaseri, H. zealandica and H. bacteriophora. Applied Soil Ecology 2007; 35: 128-139. 